intérèt à augmenter indéfiniment. le débit de l'eau sur le déversoir : Par exemple, soit une installation hydraulique où presque toute l'eau passe par les turbines ; l'eau en excès passant par dessus le barrage étant négligeable, la hauteur de chute est alors de $10 \mathrm{~m}$. Faisons arriver des quantités d'eau croissantes, les niveaux vont s'élever à l'aval plus qu'à l'amiont, la chute normale baisse. Tant que le débit par dessus le déversoir est inférieur à $460 \mathrm{~m}^{3} \mathrm{sec}$., le gain de chute par utilisation du ressaut compense à peu près exactement la baisse de chute normale. En d'autres termes, Ia turbine avale à peu près autant d'eau el conserve une puissance à peu près constante. Au delà, la charge effective $\mathrm{H}+\mathrm{J}$ diminue.

Des détails sont promis pour une publication ultérieure. Il nous a paru intéressant de signaler dès maintenant les premiers résultats acquis. Pouvons-nous exprimer le vœu que ces résultats soient bientôt contrôlés en France? Leur confirmation apporterait une sérieuse contribulion au problème de l'utilisalion des basses chutes, dont l'importance croît de jour en jour.

\title{
Quelques Considérations sur les Tôles de Conduites forcées d'Usines hydrauliques, et sur les Tôles des Chaudières.
}

\author{
Par P. DEJEAN, Docteur ès-sciences \\ Directeur du Laboratoire des essais mécaniques el métallurgiques de l'Institut Polylechnique de Grenoble'
}

\begin{abstract}
Dans une récente séance, la Société scientifique de l' lsère, après aboir éntendu la conférencé de M. Dejéth, dont nous publions le texte ci-après a émis le vou suivant:

"Etant donné l'imporlance qu'il y aurait, tant pour les constructeurs que pour les mélallurgistes, de "connaitre très exactement les conditions indispensables pour obtenir de bonnes tỏles pour conduites forcées " et pour chaudières, la Société Scientifique de l'Isère invite les intéressés à lui faire part de toutes les obser" vations que leur expérience ou leurs études systématiques ont pu leur fournir sur celte question. Elle tachera " tout particulièrement de tirer de ces renseignements les données susceptibles d'aider à la meilleure utilisation " de ces produits et à leurs conditions de réception."
\end{abstract}

Le Comité de Rédaction de la Houille Blanche s'associe à l'heureuse initiative prise par la Société Scientifique de l'Isère et informe les lecteurs de la Houille Blanche qu'elle ouvre largement les colonnes de la revue à toutes les suggestions qui pourraient lui etre communiquées sur cette importante question.

\section{Conduites Rivées. - Conduites soudées}

On sait le ròle important que joue dans l'installation d'une usine hydraulique à haute chute, la conduite forcée. Cette conduite dans laquelle circule l'eau d'alimentation des turbines, à des pressions qui peuvent atteindre 12 atmosphères et plus est constituée par une série de tronçons formés de tôles assemblées soit par soudure soit par rivure.

Analogie entre un Tronçon de conduite rivée et un Corps CyLINDRIQUe DE ChaudikrRe

Pour des raisons d'ordre technique : nécessité de résister à de brusques efforts (coups de béliers), à la dilatation et aux divers à-coups qui se produisent lorsqu'on emplit ou lorsqu'on vide les conduites, on a été amené à adopter pour la constitution des conduites métalliques un acier très pen fragile. On a choisi de l'acier extra doux dont la résistance à la rupture est comprise entre 35 et $40 \mathrm{kgs}$ par millimètre carré. La construction des tronçons rivés, tant par la nature du métal que par le mode de travail utilisé se rapproche beaucoup de celle des corps de chaudières. Il n'est donc pas illogique d'étudier simultanément ces deux genres d'appareils, si dissemblables en apparcnce : conduites rivées et corps de chaudière. Ce rapprochement ne sera pas pour surprendre du reste les consommateurs d'énergie qui rencontrent à chaque instant des points de contact bien plus inattendus entre la Houille Blanche et la Houille Noire.

\section{Conditions a exiger du Métal pour Conduites soudées}

Pour les conduites soudées, les condilions auxquelles doit répondre le métal sont un peu spéciales. Ce sont ces conditions que je vais étudier tout d'abord.

En dehors de leur résistance, élasticité et non fragilité, les tôles destinées à cette fabrication doivent avant tout être sou- dables. C'est-à-dire que les deux parties à réunir doivent après avoir été portées à une température convenable (qui est voisine de $1.300^{\circ}$ et que l'on nomme température du blanc soudant) se souder ou si l'on aime mieux adhérer parfaitement après avoir été écrasées l'une contre l'autre soit par martelage au marteau, soit au moyen de presses ou de machines spéciales, et cela sans interposition de matières étrangères.

Je n'entrerai dans aucun détail au sujet de cette soudure, je dirai simplement que deux parties bien soudées doivent donner à l'essai de traction une résistance à peu près égale (à $10 \%$ près) à celle du métal qui n'a pas été soudé.

\section{IMPORTANCE DE LA QUESTION}

Les tonnages d'une fourniture de conduile forcée importante se chiffrent par des centaines de tonnes de tôles. Si ces tòles sont défectueuses à la soudure, on voit la gravité des rebuts et les ennuis qui peuvent en résulter tant pour l'industricl qui doit fabriquer la conduite que pour le métallurgiste qui a élaboré les tôles. Il serait donc urgent d'arriver par des éludes systématiques à trouver les conditions nécessaires et suffisantes pour oblenir la soudabilité des tôles el pour en déduire les conditions indispensables à leur bonne fabrication.

\section{Soudabilité de l'Acier}

Quand on travaillait le fer fabriqué au four à puddler, cette soudure était très facile. Mais, le four à puddler avail d'aulres inconvénients, et, pour des raisons de main d'œuvre et de prix de revient, il ne se fabrique pour ainsi dire plus. Avec l'acier extra doux obtenu au four Martin ou au convertisseur, bien (que de composition très voisine de l'ancien fer, cette condition n'est pas toujours réalisée. Certaines fournitures se soudent bien 
et d'autres se soudent mal sans qu'il soit généralement possible de lo prévoir, après examen soit de la composition chimique,soit des caractéristiques du métal. Il y a donc là une anomalie que nos moyens d'investigation actuels ne sont pas susceptibles de mettre nettement en évidence.

\section{Facteurs divers susceptibles d'influencer la Soudabilité D'UN AGIER}

Une soudure peut être défectueuse, soit parce que les deux. parties que l'on cherchait à souder, bien que sans défauts apparents, ne sont pas suffisamment liées d'une manière assez intime pour pouvoir supporter des efforts analogues à ceux auxquels résiste le métal dans les autres régions; soit parce que, au cours de la soudure ou pendant le refroidissement qui la suit, il s'est produit dans la zone soudée, des criques ou fissures qui compromettent complètement la solidité de la pièce soudée. Cess denx défauts absolument distincts peuvent ètre dus à des causes très diverses, les unes sont d'ordre physique, les autres d'ordre chimique.

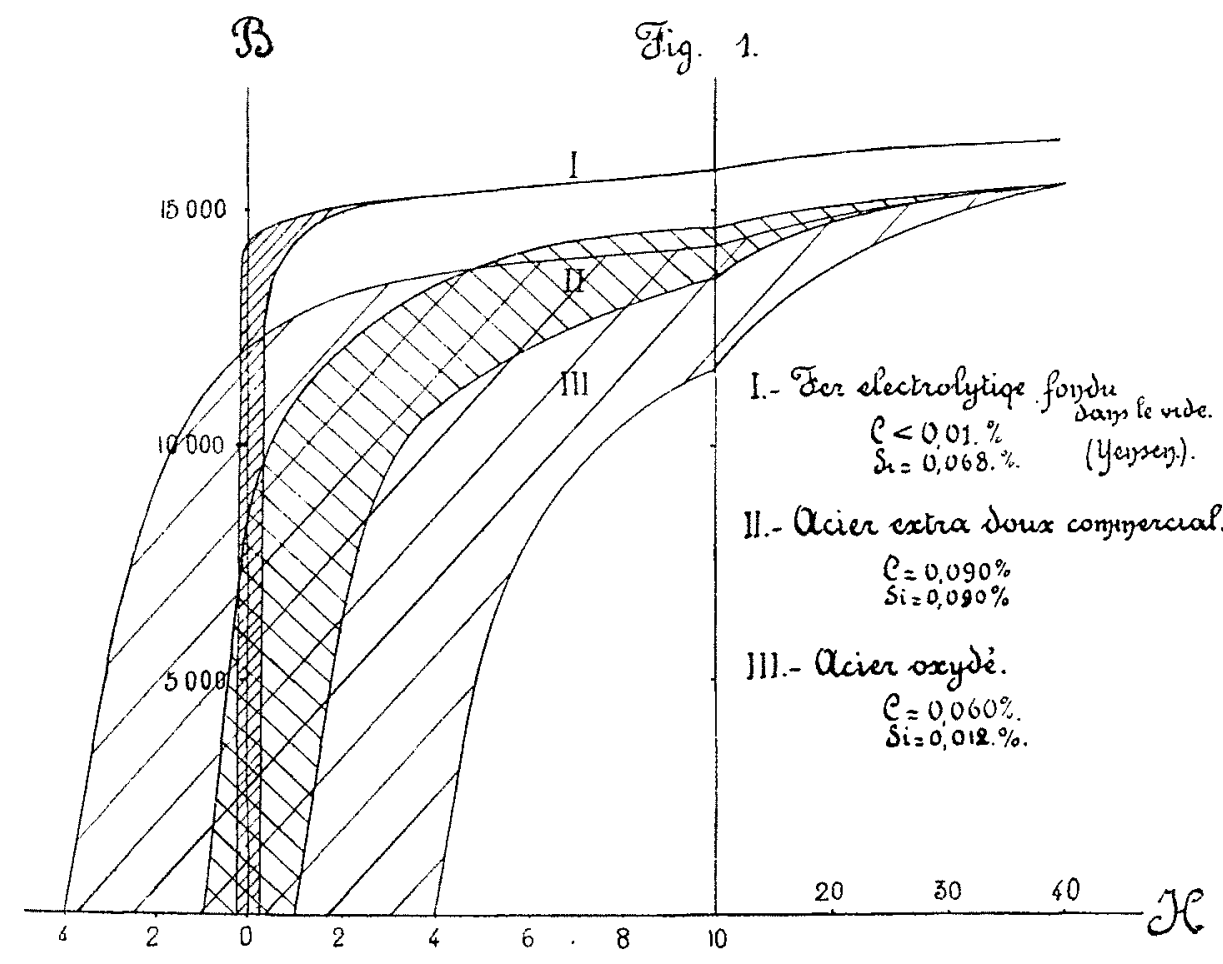

\section{FACTEURS D'ORDRE CHIMIQUE}

Sans connaître avec certitude l'influence nuisible de certains constituants chimiques sur la soudabilité, on a pu cependant tirer des nombreux essais qui ont été faits jusqu'ici, quelques présomptions sur leur nature. Nous retiendrons tout d'abord la présence de certaines impuretés : l'oxyde de fer, le soufre, le phosphore et le cuivre.

\section{OXYDATION}

On sait que l'acier est obtenu ent traitant de la fonte liquide et des riblons fondus par des oxydants qui leur enlèvent une partie de leurs impuretés, mais ces oxydants n'agissent pas seulement sur les impuretés à éliminer, ils agissent également sur le fer lui-même dont une partie se transforme en oxyde de fer et malgré la désoxydation énergique qu'on réalise avant de couler l'acier on n'arrive pas à éliminer complètement cette impureté. Or cet oxyde est partiellement soluble dans le fer non seulement à l'état liquide, mais encore lorsqu'il s'est solidifiè. De sorte qu'il en reste toujours plus ou moins dans l'acier. Comme il question. est très difficile de doser l'oxyde de fer dans l'açier, les méthodes chimiques étant extrêmement délicates et encore un peu incertaines, ce dosage est généralement laissé de côté. Ceci n'enlevant pourtant rien aux inconvénients possibles de l'oxydation. Des méthodes purement physiques pourraient permettre d'avoir une idée sur l'état d'oxydation de l'acier et parmi celles-ci nous signalerons la méthode magnétique. Un Américain, Yensen, en fondant dans le vide du fer électrolytique parfaitement désoxydé a obtenu un produit ayant une force coercitive de l'ordre de 0,225 Gauss, (tig. 1, courbe I). L'acier extra doux très bien désoxydé industriellement a une force coercitive de l'ordre de 1 Gauss (fig. 1, courbe II). Enfin, j'ai pu étudier un acier extra doux volontairement oxydé (fig. 1, courbe III) dont la force coercitive était égale à 4 Gauss. Les cycles d'hystérésie de ces trois aciers que j'ai figurés par moitié, iigure 1, ont des surfaces qui sont daris le rapport de $1 ; 5,94$ et 17. 59. Les coefficients de Steinmetz de ces trois aciers sont respectivement : 0.000265 pour l'acier (I) londu dans le vide ; 0,00157 pour l'acier (II) extra doux bien désoxydé, lype courant du commerce, et enfin de 0,00467 pour lacier (III) volontairement oxydé. On voit par là l'influence considérable de l'oxydation sur les propriétés magnétiques et l'intérêt que peuvent présenter de telles mesures pour venir en aide à l'analyse chimique.

Quelle est maintenant l'influence de l'oxydation sur la soudabilité des tôles ? La réponse à cette question est beaucoup plus délicate. On s'attache, lorsqu'on veut obtenir un acier Martin bien soudable, à avoir un métal aussi peu oxydé que pos possible, et cependant les aciers Bessemer ou Thomas mousseux (aciers vraisemblablement très oxydés) se soudent dans de bonnes conditions. Il faut noter que la corrosion se propageant très rapidement dans les aciers souffleux, on ne pourrait songer à utiliser ce métal pour la construction des chaudières, par exemple. Il faut noter aussi que l'acier oxydé présente généralement une hétérogénéité très accentuée, et que c'est à cette impureté autant qu'à la teneur en phosphore ; comme l'ont montré MM. Le Chatelier et Bogitch, qu'est due la formation, dans l'acier laminé, des lignes " ghost " qui peuvent, sous l'influence des efforts mécaniques, déterminer, des amorces de criques internes, parallèles aux faces de la tôle.

Ces simples remarques suffisent à montrer la complexité de la

\section{INFLUENCE DU SOUFRE}

Passons maintenant à l'étude du deuxième facteur que nous avons signalé : le soufre. Ce corps peut être combiné au fer ou au manganèse et former soit du sulfure de fer, soit du sulfure de manganèse. Or l'analyse chimique ordinaire nous indique bien la quantité de soufre totale contenue dans l'acier, mais elle ne nous renseigne pas sur la nature de la combinaison (notons en passant que le dosage du soufre par combustion dans l'oxygène préconisé par $M$. Travers, semble devoir donner une indication sur la proportion de ces deux sulfures). L'action du soufre est cependant très différente dans les deux cas. car le sulfure de fer est soluble dans le fer tandis que le sulfure de manganèse qui est insoluble se trouve disséminé dans lacier à l'état de petiles parcelles très fines visibles au microscope et qui rentrent dans la catégorie des scories. On admet que sous cette dernière forme (sulfure de manganèse) le soufre est beaucoup moins nuisible que le sulfure de fer qui, en se dissolvant dans le fer, peut 
en modificr beaucoup plus intimement les propriétés. C'est en particulier à ce sulfure de fer que l'on altribue à tort ou à raison le defaut dit : du rounerain sur lecuel jaurai loccasion de revenir.

\section{INFLE ExCE DU Phosphore}

Quant à la troisième impureté, le phosphore, son action sur la soudabilité est probablement beaucoup plus indirecte. (On sait que le phosphore rend l'acier fragile, mais colle fragilité se manifeste sur le métal froid el non sur le mélal porté à liaulé température. D'autre part les aciers phosphoreux présentent généralement une hétérogénéité qui se manifeste après allaque par certains réactifs appropriés, spécialement étudiés par Stead et Le Chatelier. Cette hétérogénéité prend la forme de ligne dirigées suivant le sens du laminage et auxquelles les Anglais ont domné le nom de "ghost" (fantômes). Elles sont attribuées généralement à une inégale répartition des éléments dans le métal, et bien que le phosphore semble y jouer un rôle important, il n'est probablement pas le seul coupable. Comme j'ai déjà eu l'occasion de le signaler l'oxydation du métal au moment de la coulée n'y est pas sans doute étrangère. Quoiqu'il en soil, ces lignes sont des points de faiblesse qui peuvent même déterminer dans les tôles pendant leur cintrage, ou pendant la soudure elle-même, des físsures parallèles aux faces de celles-ci.

\section{INFLteNCE DU CUIVRE}

A l'état de solution solide dans l'acier, ce corps qui est très rarement dosé, semble gèner la soudure en rendant plus difficile l'adhérence des parties à réunir. Il serait bon de fixer les limites dans lesquelles ce facteur intervient.

\section{Facteurs d'Ordre physioue}

Nous arrivons maintenant à l'étude de ce que nous arons appelé, improprement peut-être, les facteurs d'ordre physique

\section{LE Rovierain}

Ce défaut assez difficile à définir correspond à ce que l'on pourrait appeler le manque de qualilé à chaud du mélal. On l'attribue généralement à la présence du soufre et plus particulièrement, comme nous avons eu l'occasion de le signaler, à celle du sulfure de fer. Le meilleur essai pour déceler le rouverain consiste à chaufter une barre d'acier à la température du blanc soudant $\left(1300^{\circ}\right)$ puis à la laisser refroidir jusque vers $1050^{\circ}$. A cette température on essaye de la ployer. Un acier rouverain se brise alors sous le moindre choc, tandis qu'il était parfaitement plastique à une température supérieure ou à une température inférieure. Ce phénomène est du reste notablement alténué lorsqu'on commence à forger l'acier dès la température du blanc. On peut alors passer parfois la température criticue du rouverain sans aucun dommage, mais l'essai est loujours un peu risqué. Les essais de traction à chaud, effeclués en parliculier par M. Dupuy à Monlluçon sur un acier à $0,150 \%$ de carbone ont conduit à des conclusions analogues. Si on considère les variations de la striction de cet acier pour des lempéralures décroissantes à partir du blanc soudant, on constate (fig. 2) qu'il se produit vers $1050^{\circ}$ une diminution extrêmement brusque de celte striclion qui est ce qui caraclérise le mieux la plasticilé est la cohésion de l'acier. Celle variation brusque semble donc liée assez intimement avec le phénomène du rouverain dont nous venons de parler.

Les interprétations de ce phénomène, encore assez peu étudié du reste, sont assez différentes. Je signalerai simplement celle qui altribue le rouverain à une transformation possible du sulfure de fer. Quoiqu'il en soil, il est certain qu'on allénue ce défaut en ajoulant à lacier une certaine proportion de manganese. Or, on sait que edle addition facilite la formation du sulfure de manganese aux dépens du sulfure de fer. Il n'est donc pas illogicque pour les raisons précédemment indiquées d'admelle que ces deux sulfures ont des modes d'action nettement dilferents.

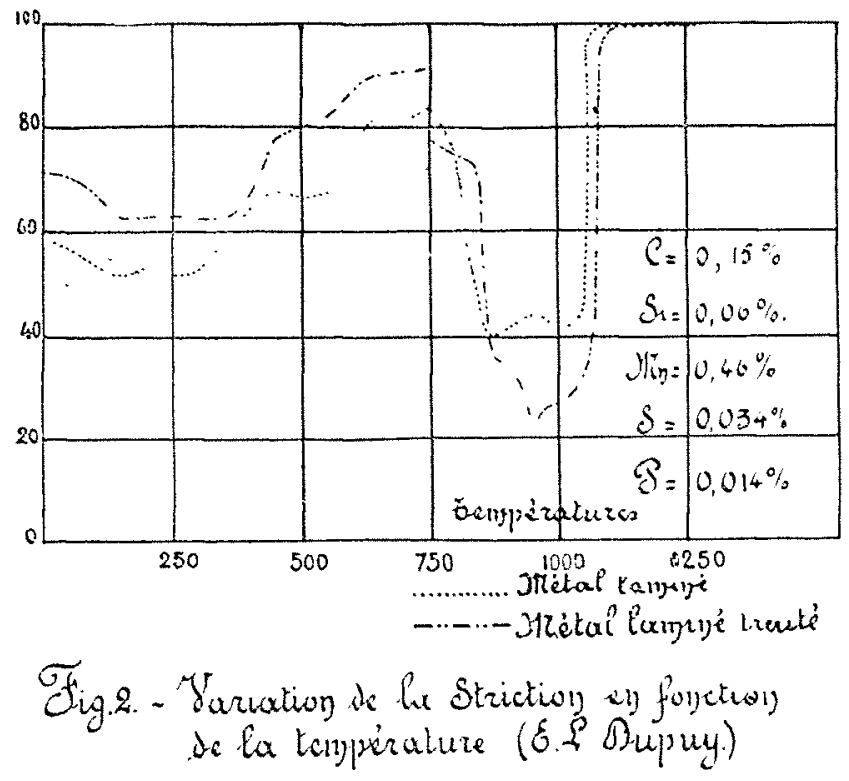

AxOMALIES DE DILATATION

Dans un important mémoire qu'il vient de présenter au Collgrès de Liège sur les conduites forcées pour usines hydrauliçues, M. Auguste Bouchayer signalait les essais entrepris sur sa demande par M. d'Aiguebelle, chef du laboratoire des Etablissements Bouchayer el Viallet, à Grenoble. Il ressort de ces essais que la soudabilité es en relation trés étroile avec l'anplitude de l'anomalic de dilatalion qui se produit au point $A_{*}$ Si on appelle $\downarrow_{m}$ la contraction des ordonnées de la courbe "dilatation-temperature" (fig. 3) qui se produit an cours de cette anomalie, on constate par exemple qu'un acier ayant un $\lrcorner_{\mathrm{m}}$ de l'ordre de 0,17 à $0,18^{\circ} \%$ (fig. 3 courbe 1) se soude très mal, alors qu'un autre dont le $\perp$ m est de l'ordre de 0,05 à $0,06 \%$ (fig. 3 courbe 2) se soude bien. Il est nécessaire d'ajouter que Ia mauvaise qualité de la première, soudure n'est pas tant due au manque d'adhérence des deux parlies soudées l'une sur l'autre qu'aux criques qui se rencontrent sur la partie soudé ou à son voisinage. Les lensions occasionnées par l'anomalic de dilatation qui ne se produil pas au mente moment dans les différentes parties de la soudure pourraient expliquer ce résullat. Lorsque le passage au point $A_{; ;}$est accomgné d'une brusque dilatation. (coube 1), les criques ont une bien plus grande lendance à se produire que lorsque l'anomalie est élalée sur un grand intervalle de Lempérature el que son auplitude est plus faible (courbe 2). Quoiçu'il en soit, M. Bouchayer énonce la règle suivante : "Un acier exlra-doux ayant une " somme d'impuretés variant de 0,6 à $0,9 \%$ se soude d'autanl " mieux que son anomalie moyenne de dilatation $\lrcorner_{m}$ est "moindre.

"L'expérience montre que dans ces limiles de composilion, $J_{m}$ "varic de $0,05 \%$ à $0,19 \%$ constiluant un réctif particuliert"ment sensible des propriélés du mélal chudić ".

Il ajoute en outre : "Ces comparaisons cl regles no sont vala* bles que pour les mênes alliages, car lo ler pur donne un dia- 
gramme encore plus, exagéré que la conrbe No 1 el un $\Delta_{m}$ egul it $0,282 . "$

\section{Courbe $) \stackrel{0}{=} 1$.}

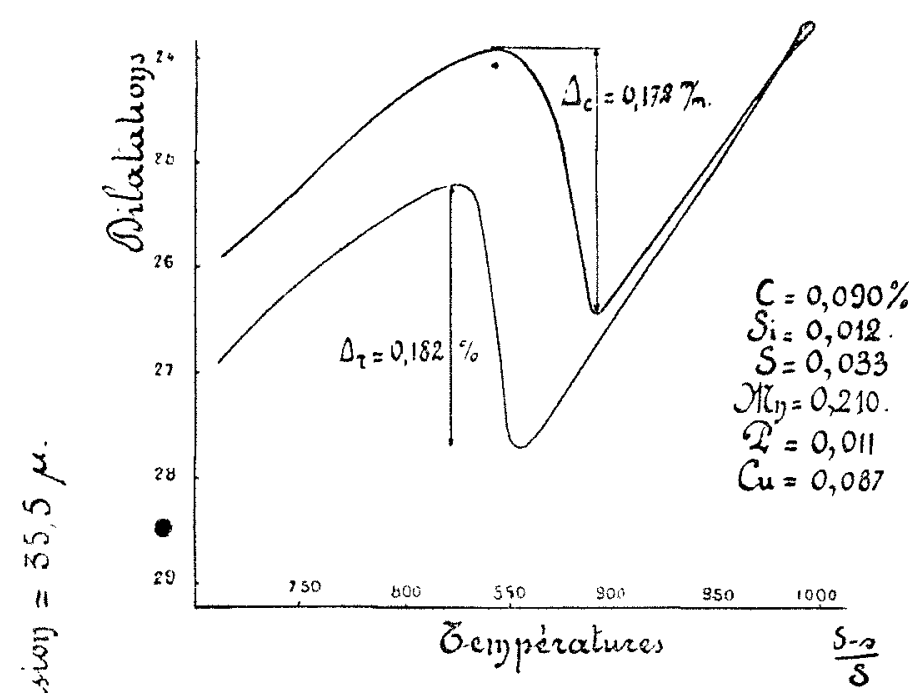

\section{Courbe $)(\because 2$}

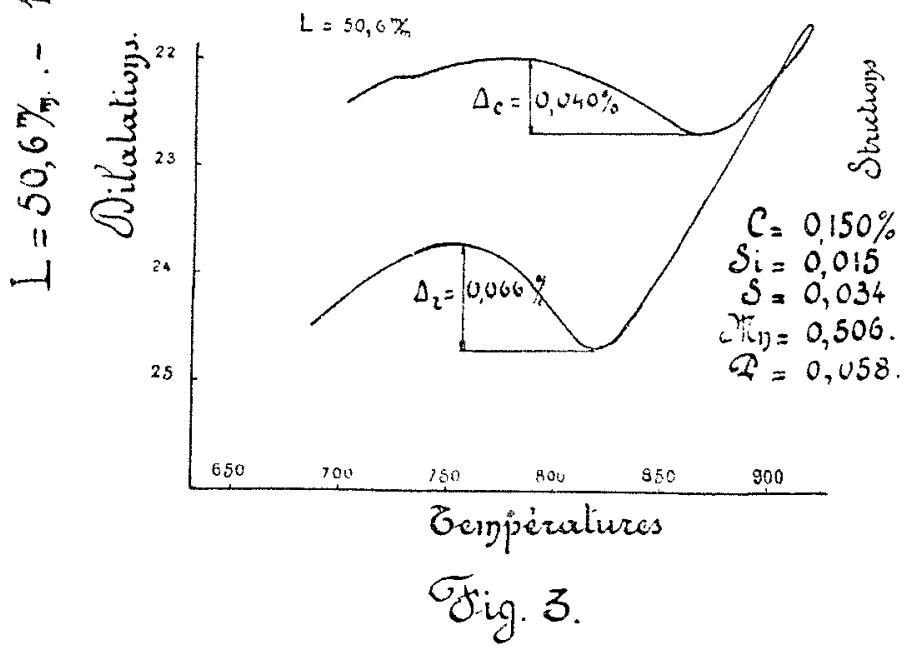

Conclusions Relatrves a L'ÉTtde de la SOUdAbILtét

J'ai lout d'abord cherche à montrer l'influence qu'exerce sur la soudabilité un certain nombre de facteurs d'ordre chimique.

l'armi jes premiers jai indique le soufre, dingereux surtout par la formation da sulfure de fer, el dont l'influence néfaste semhls: allénuée si on facilite par des addilions de manganèse son passage à l'élat de sulfure de manganèse.

le phosphore, lui, dome lieu surlont dans un métal oxydé, à une bélérogénéilé qui se manifeste par la formation de lignes paralleles an sens de laminage, qui sont autant de points faibles susceptibles d'amorcer des criques.

parmi les phénomènes physiques qui semblent jouer un certain rôte dans les difficultés que l'on rencontre au cours de la soudure j'ai signalé : le Rouwerain el l'anomalie de dilatulion.

Il est bien culendu que loul ce qui a élé dit ne s'applique quaux aciers extra-doux, les seuls susceptibles de se souder par forgenge.

Je n’ai pas la prélention d'avoir épuisé la question ni même d'atvoir domné une juée d'ensemble de son élat actuel. J'espère loutelois que les quelques détails que jai fournis suftiront à en montrer la complexilé, et combien l'élat de nos connaisssances sur ce poinl laisse encore à désirer. Je crois qu'il y aurait intérèt à rassembler toutes les observations qui ont été faites sur lout ce qui Louche à la soudabilité, tant dans les ateliers qui pratiquent ce genre de soudure, que dans les usines métallurgiques qui fabriquent les tôles. La chose en vaul la peine.

Le procédé de soudure serail encore bien plus intéressanl si l'on pouvait y adapter non seulement de l'acier exlra-doux, mais aussi un acier plus dur, permettant d'obtenir des conduites plus résistantes et moins pesantes. Cette question serait très importante à étudier.

\section{Conduites RIVEES ET CORPS DE CHAUdiÉRE}

La question des tôles pour conduites rivées et pour corps de chaudière semble beaucoù plus simple et je m'y étendrai beaucoup moins.

Le travail de rivure ne nécessitant pas le chauffage des tôles. Nous n'avons pas à nous inquiéter des conditions de travail à chaud,; mais le travail à froid à d'autres inconvénients, en particulier l'écrouissage qui tend à rendre le métal fragile dans la région martelée. En outre, les conduites comme les chaudières peuvent être appelées à subir des à-coups spécialement par suite des coups de béliers, aussi bien qu'au moment de l'emplissage et de la vidange de la conduite, d'autre part, comme elles doivent résister aux déformations provenant des dilatations, le métal employé doit être aussi peu fragile que possible et cetle condition n'est réalisée qu'avec un métal très doux : 30 à 35 kilogs de résistance.

Pour les chaudières, les compagnies de chemins de fer envisagent deux nuances :

A) nuance "extra douce" $\mathrm{R}=35 \mathrm{Kgs} \mathrm{A} \%=30$ sous $\mathrm{L}=V \overline{66,67 \mathrm{~S}}$ pour tôles de boîtes à feu.

B) nuance « douce ».. $\mathrm{R}=40 \mathrm{Kgs}: \mathrm{A} \%=27$ sous $\mathrm{L}=V \overline{66,67 \mathrm{~S}}$ pour corps cylindriques.

Le cahier des charges "Véritas " (chaudières des navires) ra beaucoup plus haut dans les résistances qu'il impose.

$$
\begin{aligned}
& \mathrm{R}=38 \text { à } 46 \mathrm{Kgs} \text { pour foyers et boîtes à feu. } \\
& \mathrm{R}=40 \text { à } 48 \mathrm{Kgs} \text { pour fonds et façades. } \\
& \mathrm{R}=42 \text { à } 50 \mathrm{Kgs} \text { pour viroles. }
\end{aligned}
$$

Certaines tôles de coup de feu doivent toujours être constiluées par du mélal extra doux de manière à écarter les effets de trempe par l'eau d'alimentation lorsque, par suite de négligence dans la conduite du générateur, des tôles ont accidentellcment alleint une température élevée.

Cette préoccupation de la trempe se retrouve dans le cahicr des charges de compagnies de chemin de fer qui impose le ployage à bloc après trempe

Le cahier des charges de "l'Association Lyomnaise des Propriétaires d'appareils à vapeur " avait une dispositicn analogue.

Des avenants ont modifié provisoircment ces dernières clauses. Il serait intéressant de savoir quel en est le bien fondé réel.

Enfin, la tempéralure de régime de corlaines tôles de chaudieres est voisine de $210^{\circ}$. Or on sait qu'on s'approche ainsi d'une température crilique pour l'acier qui présente alors ce que l'on a désigné sous le nom de "fragilité au bleu ". Celte fragilité peut faciliter l'amorçage des fissurations dans les parties soumises à des efforts successifs de contraction el de dilatation.

Ces quelques considérations suftisent à montrer que, malgré sa simplicité apparenle, cette question encore aurait besoin d'ètre éclaircie pour arriver à une entente et à lunification des clauses des cahiers des charges. Les avis molivés des métallurgistes, des construcleurs et des usagers seraient très intéressants à recueillir. 